\title{
Low-intensity treadmill exercise protects cognitive impairment by enhancing cerebellar mitochondrial calcium retention capacity in a rat model of chronic cerebral hypoperfusion
}

\author{
Jae-Min Lee ${ }^{1, \dagger}$, Jongmin Park ${ }^{2,}$, Joo-Hee Lee ${ }^{1}$, Hyo-Bum Kwak ${ }^{3}$, Mi-Hyun No ${ }^{3}$, Jun-Won Heo ${ }^{3}$, Youn-Jung Kim ${ }^{1, *}$ \\ 'Department of Basic Nursing Science, College of Nursing Science, Kyung Hee University, Seoul, Korea \\ ${ }^{2}$ Research Institute of Nursing Science, College of Nursing, Pusan National University, Yangsan, Korea \\ ${ }^{3}$ Department of Kinesiology, Inha University, Incheon, Korea
}

Chronic cerebral hypoperfusion (CCH) is caused by reduced blood flow to the brain representing gradually cognitive impairment. CCH induces mitochondrial dysfunction and neuronal cell death in the brain. Exercise is known to have a neuroprotective effect on brain damage and cognitive dysfunction. This study aimed to clarify the neuroprotective effect of low-intensity treadmill exercise (LITE) by enhancing cerebellar mitochondrial calcium retention capacity in an animal model of CCH. Wistar rats were divided into the sham group, the bilateral common carotid arteries occlusion (BCCAO) group, and the BCCAO and treadmill exercise $(\mathrm{BCCAO}+\mathrm{Ex})$ group. $\mathrm{BCCAO}+\mathrm{Ex}$ group engaged the LITE on a treadmill for 30 min once a day for 8 weeks before the BCCAO surgery to investigate the protective effect of LITE on cognitive impairment. CCH induced by $\mathrm{BCCAO}$ resulted in mitochondrial dysfunction in the cerebellum, in- cluding impaired calcium homeostasis. CCH also decreased cerebellar Purkinje cells including of calbindin D28k and parvalbumin, resulting in cognitive impairment. The impairment of mitochondrial function, loss of cerebellar Purkinje cells, and cognitive dysfunction ameliorated by exercise. The present study showed that LITE hindered the deficit of spatial working memory and loss of Purkinje cell in the cerebellum induced by CCH. We confirmed that the protective effect of LITE on Purkinje cell by enhanced the mitochondrial calcium retention capacity. We suggest that LITE may protect against cognitive impairment, and further studies are needed to develop the intervention for patients who suffered from $\mathrm{CCH}$.

Keywords: Exercise, Cerebral hypoperfusion Cognition, Cerebellum, Mitochondria, Calcium

\section{INTRODUCTION}

Chronic cerebral hypoperfusion (CCH), the main reason for vascular dementia $(\mathrm{VaD})$, is caused by the thromboembolic events of the brain, results in progressive cognitive impairment (Iadecola, 2013). The cerebral cortex, hippocampus, and white matter are susceptible to hypoperfusion-induced lesions. A reduction of cerebral blood flow leads to hypoxia and oxidative stress. The hypoxia-induced oxidative stress causes mitochondrial dysfunction and neuronal cell death via the release of reactive oxygen species (Ven- kat et al., 2015). Although it is essential to understand the pathophysiology of the $\mathrm{CCH}$ for establishing the proper animal model to develop treatments, the pathology and mechanism of $\mathrm{CCH}$ have not yet been fully understood. Hence, it needs a new approach to understand the mechanism better.

The role of the cerebellum has recently been reestablished to represent a cognitive function. The description of the cerebellar cognitive affective syndrome (CCAS) and topographical study of the cerebellum indicate the cerebellar engagement into neural circuits relevant to cognition and emotion (Jacobs et al., 2018). CCAS

\footnotetext{
*Corresponding author: Youn-Jung Kim (DD https://orcid.org/0000-0003-4991-0825 Department of Basic Nursing Science, College of Nursing Science, Kyung Hee University, 26 Kyunghee-daero, Dongdaemun-gu, Seoul 02447, Korea Email: yj129@khu.ac.kr

†These authors contributed equally to this study as co-first authors.

Received: August 12, 2021 / Accepted: September 18, 2021
}

This is an Open Access article distributed under the terms of the Creative Commons Attribution Non-Commercial License (https://creativecommons.org/licenses/by-nc/4.0/) which permits unrestricted non-commercial use, distribution, and reproduction in any medium, provided the original work is properly cited. 
signifies a deficit in the cognitive domain of executive function, spatial memory, and language caused by the cerebellar damage (Schmahmann and Sherman, 1998). The cerebellum is mainly related to cognitive function, especially related to spatial navigation, situated in the posterior lobe of the cerebellum. Damage to the posterior lobes of the cerebellum is associated with impairment of spatial working memory and knockout mice with cerebellar dysfunction have been shown to exhibit spatial navigation disorders (Tomlinson et al., 2014). Our previous study was confirmed that the loss of Purkinje cell that has contained plenty of calciumbinding proteins in the posterior lobe caused by $\mathrm{CCH}$ affects spatial navigation impairment (Lee et al., 2018).

It has been reported that the loss of Purkinje cells in the cerebellum is caused by glutamate release and excitotoxicity due to intracellular calcium imbalance (Welsh et al., 2002). Intracellular calcium homeostasis is vital in maintaining calcium-related signaling pathways and nerve function in healthy brains (Pchitskaya et al., 2018). Calcium channel, endoplasmic reticulum, calciumbinding protein, and mitochondria perform a buffer system to prevent calcium overload. Mitochondria are essential calcium modulators that absorb calcium to reduce cellular calcium levels. Mitochondria are regulating synaptic transmission, brain function, and cognitive function. Mitochondrial dysfunction has been found to be involved in the pathology of neurodegenerative diseases such as amyotrophic lateral sclerosis, Alzheimer disease, and Parkinson disease (Marambaud et al., 2009). Many diseases impaired neuroplasticity, which leads to cell dysfunction, or even cell death. Recently previous study, exercise strengthened mitochondria in the brain by increasing neuroplasticity and suppressing cell death (Seo et al., 2019).

Enhancing mitochondrial function may be a therapeutic approach to prevent the loss of Purkinje cells. It is a well-known intervention that treadmill exercise improves cognitive function by reducing the neuronal apoptosis and the responsiveness of astrocytes in the cerebellum following global ischemia and attenuates age-related Purkinje cell loss (Larsen et al., 2000; Seo et al., 2010). The study aimed to examine whether $\mathrm{CCH}$ induces a loss of Purkinje cells, and to investigate the effect of low-intensity treadmill exercise (LITE) on cognitive function by enhancing the mitochondrial calcium retention capacity in the cerebellum.

\section{MATERIALS AND METHODS}

\section{Animals}

We used adult male Wistar rats (body weight $80 \pm 10 \mathrm{~g}, 4$ weeks old) in this experiment. We randomly divided the rats into three groups: the sham group $(n=8)$, the bilateral common carotid arteries occlusion (BCCAO) group $(n=8)$, and the BCCAO and treadmill exercise $(\mathrm{BCCAO}+\mathrm{Ex})$ group $(\mathrm{n}=8)$. All animals were inhabited under controlled conditions of temperature $\left(25^{\circ} \mathrm{C} \pm 2^{\circ} \mathrm{C}\right)$, humidity (50\%-55\%), and light (12-hr light-dark cycles), with access to food and water ad libitum. All animal experimental procedures were approved by the National Institutes of Health and the guidelines of the Korean Academy of Medical Science. This study obtained approval by the Institutional Animal Care and Use Committee of Kyung Hee University (KHUASP [SE]-17-138).

\section{Bilateral common carotid arteries occlusion}

We anesthetized the rats in 3\% halothane in fresh gas flow composed of an $\mathrm{N}_{2} \mathrm{O}: \mathrm{O}_{2}$ (70:30). A ventral right incision in the neck was performed to expose the right common carotid artery, which was carefully separated to avoid damage of surrounding tissues particularly near a vagus nerve. The right common carotid artery was ligated doubly with 3-0 silk (Ailee, Seoul, Korea) below the carotid bifurcation. A left common carotid artery occlusion was performed in the same procedure at 1 week after the right common carotid artery surgery. Sham-operated animals underwent the same operation process without the vessel ligation.

\section{LITE protocol}

Before the surgery, the rats were engaged on a treadmill for 30 min for 8 weeks, according to the previously described method (Heo et al., 2014). The LITE procedure was made up running at $2 \mathrm{~m} / \mathrm{min}$ for the first of $5 \mathrm{~min}$, at $3 \mathrm{~m} / \mathrm{min}$ for the next $5 \mathrm{~min}$, and then at $5 \mathrm{~m} / \mathrm{min}$ for the last $20 \mathrm{~min}$ at $0^{\circ}$ of inclination. The rats in the nonexercise groups were put in the treadmill without running for the same period as the exercise group.

\section{Radial arm maze test}

The radial 8-arm maze test was performed to assess spatial working memory ability after 3 weeks of BCCAO. The radial 8-arm maze consists of eight equally spaced arms $(60 \mathrm{~cm} \times 12 \mathrm{~cm} \times 12 \mathrm{~cm})$ radiating from a central octagonal platform, placing the rat on the central octagonal platform and collecting water hidden at the tip of the arm. On the first day of training, rats can enter each arm arbitrarily to collect water hidden in each arm for $8 \mathrm{~min}$. After $24 \mathrm{hr}$, the test day scores the arm selection when all four paws of the rat into the arm. On test day after $24 \mathrm{hr}$, rats will go into each arm to find water, and revisiting a previously visited arm will result in an error record. The number of correct selections before the first error was 
measured, and the error was counted when they revisited the arms.

\section{Immunohistochemistry}

Serial sagittal sections of $40-\mu \mathrm{m}$ thickness were obtained using a freezing microtome (Leica, Nussloch, Germany). Immunohistochemistry was performed to investigate the expression of calbindin D28k and parvalbumin. Free-floating sections were initially incubated in $3 \% \mathrm{H}_{2} \mathrm{O}_{2}$ for $30 \mathrm{~min}$ at room temperature. After being blocked with $10 \%$ normal horse and rabbit serum for $1 \mathrm{hr}$, the sections were incubated overnight at $4^{\circ} \mathrm{C}$ with calbindin $\mathrm{D} 28 \mathrm{k}$ antibody (1:500; Abcam, Cambridge, UK), and parvalbumin antibody (1:1,000; Abcam). The sections were then incubated for $2 \mathrm{hr}$ with the biotinylated rat and mouse secondary antibody (1:200; Vector Laboratories, Burlingame, CA, USA) for $1 \mathrm{hr}$ at room temperature. The bound secondary antibody was then amplified using a Vector Elite ABC kit (Vector Laboratories) for $1 \mathrm{hr}$ at room temperature. For staining, the sections were incubated in a solution consisting of $0.02 \%$ diaminobenzidine and $0.03 \% \mathrm{H}_{2} \mathrm{O}_{2}$ in $50 \mathrm{mM}$ Tris- $\mathrm{HCl}(\mathrm{pH}, 7.6)$ for approximately $5 \mathrm{~min}$, following which they were washed with phosphate-buffered saline and mounted onto gelatin-coated slides. Cover slips were mounted using Permount (Fisher Scientific, Waltham, MA, USA). The number of positive cells per section was counted in 5 random fields from every specimen with a Nikon Eclipse 80i microscope (magnification, $\times 40$; Nikon Corporation, Tokyo, Japan).

\section{Mitochondrial calcium retention capacity}

When the mitochondria are no longer able to retain calcium, calcium ion $\left(\mathrm{Ca}^{2+}\right)$ exit the mitochondria through the permeability transition pore (PTP) opening of the mitochondria, we can

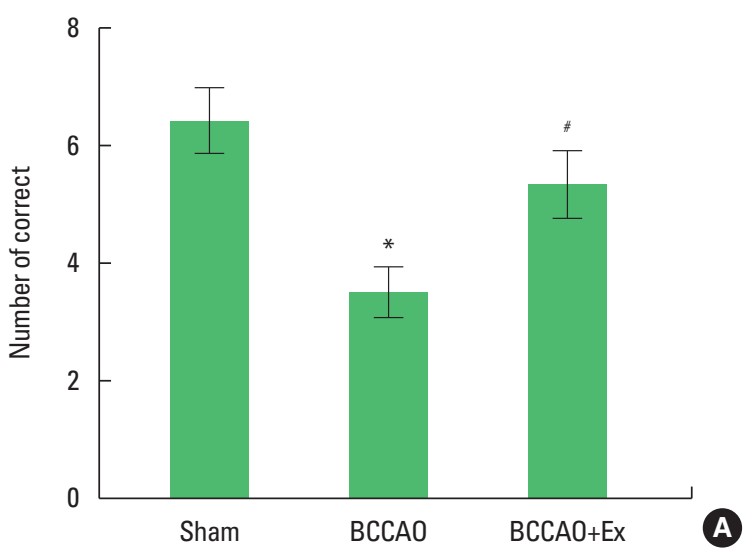

measure the $\mathrm{Ca}^{2+}$ retention capacity by the susceptibility to mitochondrial PTP opening. After homogenizing the cerebellum tissue, we continuous measured response of calcium green 5-N fluorescence using a Spex Fluoromax 4 (HORIBA, Edsion, NJ, USA) at $37^{\circ} \mathrm{C}$ state four respiration $(10-\mu \mathrm{g} / \mathrm{mL}$ oligomycin). After setting the background, and then $\mathrm{Ca}^{2+}(12.5 \mathrm{nM})$ was injected periodically at $506 \mathrm{~nm}$ (excitation wavelength) and $532 \mathrm{~nm}$ (emission wavelength) to measure and analyze the reaction. The maximum $\mathrm{Ca}^{2+}$ uptake capacity of the mitochondria before PTP opening $\left(\mathrm{Ca}^{2+}\right.$ release) was represented as picomoles/sec/mg tissue weight.

\section{Statistical analysis}

We expressed data mean \pm standard error of the mean. IBM SPSS Statistics ver. 25.0 (IBM Co., Armonk, NY, USA) was used for statistical analysis. Statistical analysis was performed using one-way analysis of variance followed by Tukey post hoc test. Differences between groups were regarded as significant at $P<0.05$.

\section{RESULTS}

\section{Protected cognitive performance}

The radial arm maze test was used to confirm the establishment of $\mathrm{VaD}$ animal model and evaluate the effect of low-intensity exercise on the cognition dysfunction induced by $\mathrm{CCH}$. We verified the well-established animal model through the results that the BCCAO group (3.5 \pm 0.4$)$ was decreased correct performance compared to the sham group $(6.4 \pm 0.5)(P<0.05)$ (Fig. 1A). BCCAO+ Ex group (5.3 \pm 0.6$)$ showed significantly better performance as compared with the BCCAO group $(P<0.05)$ (Fig. 1A). Error performance also showed the same result (Fig. 1B). We confirmed

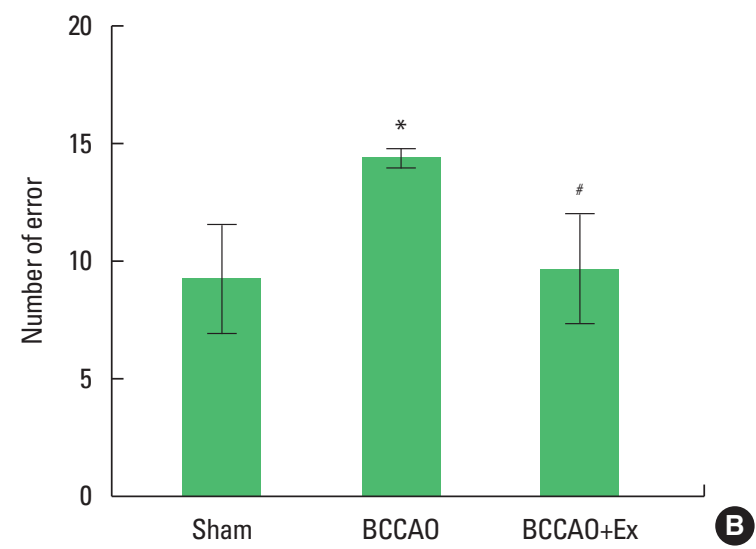

Fig. 1. Effect of low-intensity treadmill exercise on spatial working memory. (A) Corrects measured prior to the first error. (B) Errors were measured when rats revisited the arms. The results are presented as the mean \pm standard error of the mean. ${ }^{*} P<0.05$ compared with sham group. ${ }^{*} P<0.05$ compared with bilateral common carotid arteries occlusion group. Sham, sham group; BCCAO, bilateral common carotid arteries occlusion group; BCCAO+Ex, BCCAO and treadmill exercise group. 

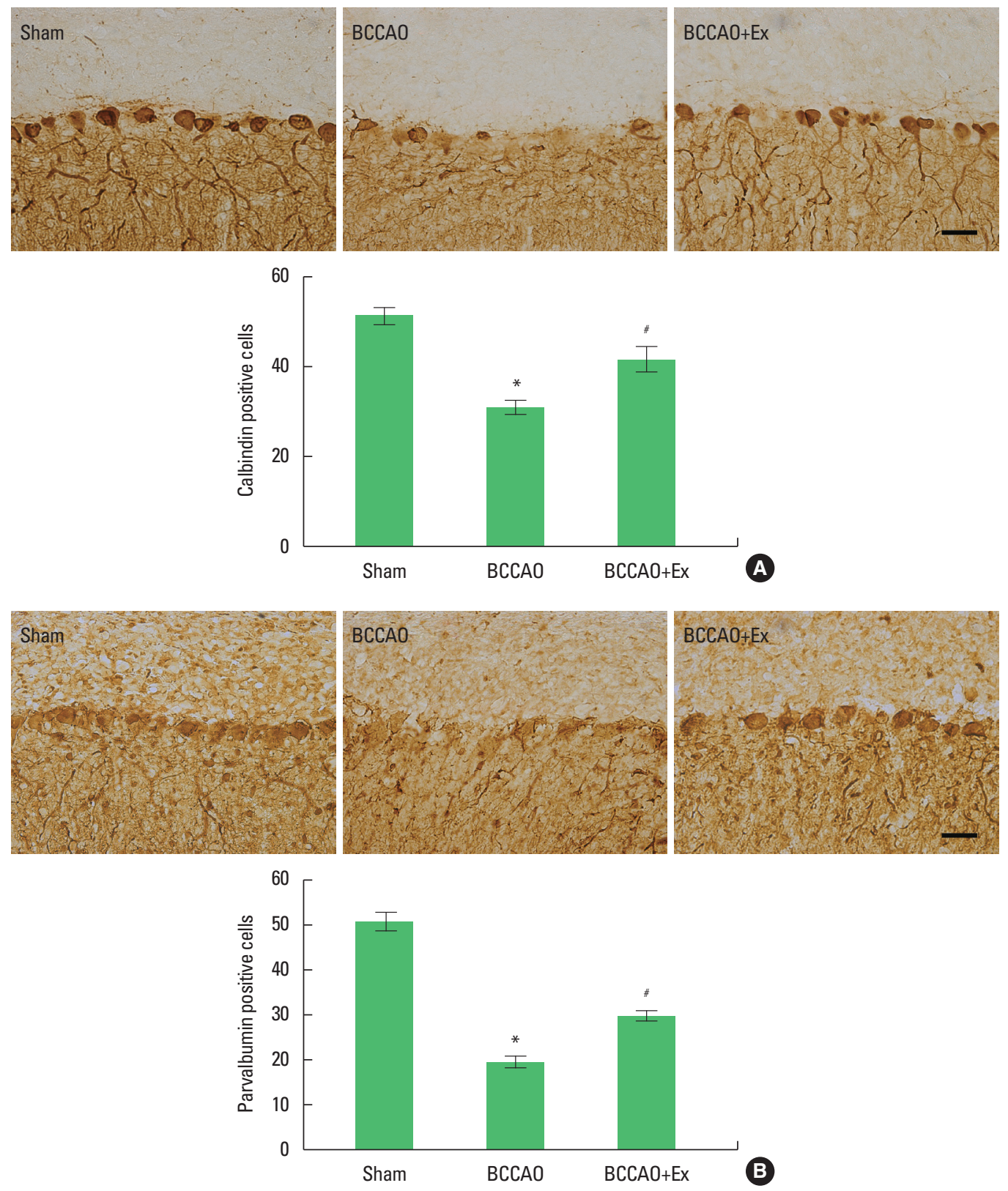

Fig. 2. Effect of low-intensity treadmill exercise attenuates Purkinje cell loss induced by chronic cerebral hypoperfusion in the posterior lobe of the cerebellum. (A) Calbindin D28K positive cells. Upper panel: photomicrographs of immunostaining of calbindin D28k positive cells. The scale bars represent 50 m. Lower panel: number of calbindin D28k positive cells. (B) Parvalbumin-positive cells. Upper panel: photomicrographs of immunostaining of parvalbumin-positive cells. The scale bars represent $50 \mu \mathrm{m}$. Lower panel: number of parvalbumin-positive cells. The results are presented as the mean \pm standard error of the mean. ${ }^{*} P<0.05$ compared with sham group. ${ }^{\sharp} P<0.05$ compared with bilateral common carotid arteries occlusion group. Sham, sham group; BCCAO, bilateral common carotid arteries occlusion group; $\mathrm{BCCAO+Ex,} \mathrm{BCCAO} \mathrm{and} \mathrm{treadmill} \mathrm{exercise} \mathrm{group.}$

the improved cognitive performance by LITE.

\section{Decreased loss of Purkinje cell}

Calbindin D28k and parvalbumin are proteins that are participated in $\mathrm{Ca}^{2+}$ signaling, protects neuronal cells through reduced intracellular $\mathrm{Ca}^{2+}$ concentrations to maintain $\mathrm{Ca}^{2+}$ homeostasis, mainly locate in Purkinje cells and molecular layer interneurons in the cerebellum. The number of calbindin-positive Purkinje cells in the BCCAO+Ex group (41.50 \pm 2.66$)$ was also significantly higher than the $\mathrm{BCCAO}$ group $(31.00 \pm 1.67)(P<0.05)$ (Fig. 2A). 

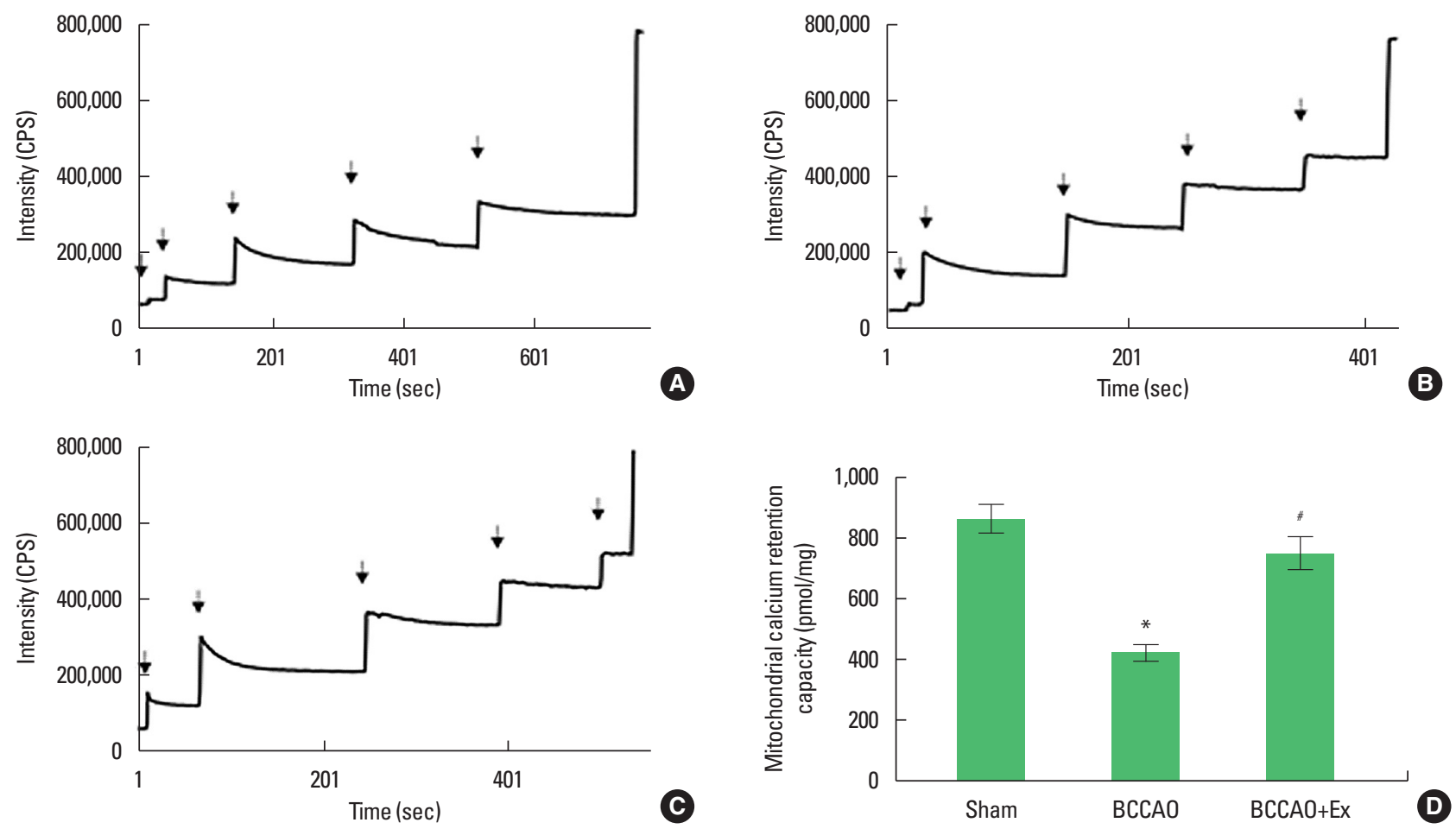

Fig. 3. Effect of low-intensity treadmill exercise on mitochondrial calcium retention capacity in the cerebellum. (A) Sham group. (B) Bilateral common carotid arteries occlusion group. (C) Bilateral common carotid arteries occlusion and treadmill exercise group. (D) Quantified total mitochondrial calcium retention capacity. Experimental traces representing increases in calcium green $5-\mathrm{N}$ fluorescence in response to $1 \mu \mathrm{M} \mathrm{Ca}^{2+}$. The results are presented as the mean \pm standard error of the mean. ${ }^{*} P<0.05$ compared with sham group. ${ }^{\sharp} P<0.05$ compared with bilateral common carotid arteries occlusion group. CPS, counts per second; Sham, sham group; $\mathrm{BCCAO}$, bilateral common carotid arteries occlusion group; $\mathrm{BCCAO+Ex,BCCAO}$ and treadmill exercise group.

The BCCAO+Ex group $(29.83 \pm 1.01)$ showed significantly increased the number of parvalbumin-positive Purkinje cells compared with the BCCAO group $(19.67 \pm 1.43)(P<0.05)$ (Fig. 2B). These results indicate that LITE protects the neuronal cell viability in the cerebellum.

\section{Enhanced mitochondrial calcium retention capacity}

One of major role of mitochondria is retention of calcium when they exceed the normal range of intracellular calcium level. In our study, the BCCAO+Ex group (751.28 \pm 52.97$)$ showed significantly increased calcium retention capacity compared with the BCCAO group (424.19 \pm 28.46$)(P<0.05)($ Fig. 3). This result shows that low-intensity exercise has protective effect in the cerebellum by increasing calcium retention capacity.

\section{DISCUSSION}

The present study demonstrated that LITE protects the cognitive impairment induced by $\mathrm{CCH}$ through neuronal cell viability in the cerebellum and increasing the cerebellar mitochondrial cal- cium-binding capacity. The cerebellum has emerged a critical role in cognitive function as a connection between cerebrum and cerebellum, including cerebello-thalamo-cortical and cortico-pontocerebellar pathways (Palesi et al., 2017). It has been hypothesized that dysfunction of the cerebellum may play an important role in the pathology of $\mathrm{VaD}$ (Sui and Zhang, 2012). However, little research has been conducted in the cerebellum compared to the other brain regions to uncover the mechanism of $\mathrm{CCH}$. The increase in studies of the cerebellum will be an opportunity for us to establish an essential basis for developing interventions that protect or restore cognitive health.

Most subjects who have suffered from $\mathrm{CCH}$ are old aged, so it is necessary to develop feasible and safe interventions for frail elderly (Buchner and Coleman, 1994). In this study, we applied the LITE to the $\mathrm{CCH}$ animal model. The low-intensity exercise showed a maximum oxygen uptake of $54 \%$ to $58 \%$ and better exercise adherence compared to the high-intensity exercise (Schefer and Talan, 1996). Moreover, the exercise reduced in injury risk and was generally more easily accessible to older adults (Marques-Aleixo et al., 2015). We observed the LITE prevented impaired cognitive 
performance induced by $\mathrm{CCH}$. These results are consistent with previous studies showed that treadmill exercise ameliorated spatial memory performance using the 8-arm maze and Morris water maze test induced by CCH (Lee et al., 2017; Lee et al., 2018).

A previous study found that treadmill exercise in $\mathrm{CCH}$-induced animal models has a preventive effect on the loss of Purkinje cells in the cerebellum via the suppression of glial cells and apoptosis (Lee et al., 2018). Our study enhanced the mitochondrial calcium retention capacity by LITE protected the loss of Purkinje cell in the cerebellum. Diminished Purkinje cells showed the spatial working memory deficit by acting minor or indirect role (Martin et al., 2004). Purkinje cells also express plenty of calcium-binding proteins, such as calbindin-D28k and parvalbumin. These proteins have engaged the regulation of cell cycle and intracellular calcium concentration related to apoptosis (Zhao et al., 2008). One of the main causes of loss of Purkinje cell has been known the mitochondrial dysfunction.

Mitochondrial dysfunction is regarded as one of the major causes of neuronal injury in $\mathrm{VaD}$ induced by $\mathrm{CCH}$ (Venkat et al., 2015). Dysfunction of mitochondria represents diminished production of adenosine triphosphate, impaired calcium buffering capacity, and increased reactive oxygen species (Zorov et al., 2014). The mitochondrial PTP and forms a transmembrane pore that is large enough to allow release of cytochrome c. Mitochondrial calcium retention capacity was checked by measuring the PTP opening of mitochondria. Notably, the decline of calcium retention capacity affects the mitochondrial calcium homeostasis, which results in apoptosis through the caspase signaling cascade (Hajnóczky et al., 2006). Intracellular calcium overload activates the caspase that the proteolytic activity of caspases has the biochemically vital role of apoptosis (Zhivotovsky and Orrenius, 2011). In this study, we observed the mitochondrial calcium retention capacity in $\mathrm{VaD}$ group was decreased compared to sham group. Exercise improved the mitochondrial calcium retention capacity in the brain (Seo et al., 2019). Especially, low-intensity exercise improved physical and cognitive health (Tse et al., 2015). In accord with our result, the previous research showed that physical exercise increased manganese-dependent superoxide dismutase activity and calcium retention capacity in the cerebellum (Marques-Aleixo et al., 2015).

In conclusion, we showed that LITE might exert a neuroprotective effect against the loss of Purkinje cells via increasing the mitochondrial calcium retention capacity in the cerebellum. Therefore, LITE may have potential as a therapeutic intervention strategy for the attenuation of cognitive impairment in patients with $\mathrm{CCH}$.

\section{CONFLICT OF INTEREST}

No potential conflict of interest relevant to this article was reported.

\section{ACKNOWLEDGMENTS}

This study was supported by the Ministry of Education of the Republic of Korea and the National Research Foundation of Korea (NRF-2016S1A5B5A01022873 and NRF-2019R1I1A1A01060991).

\section{REFERENCES}

Buchner DM, Coleman EA. Exercise considerations in older adults: intensity, fall prevention, and safety. Phys Med Rehabil Clin N Am 1994;5: 357-375.

Hajnóczky G, Csordás G, Das S, Garcia-Perez C, Saotome M, Roy SS, Yi M. Mitochondrial calcium signalling and cell death: Approaches for assessing the role of mitochondrial $\mathrm{Ca}^{2+}$ uptake in apoptosis. Cell Calcium 2006;40:553-560.

Heo YM, Shin MS, Kim SH, Kim TW, Baek SB, Baek SS. Treadmill exercise ameliorates disturbance of spatial learning ability in scopolamine-induced amnesia rats. J Exerc Rehabil 2014;10:155-161.

Iadecola C. The pathobiology of vascular dementia. Neuron 2013;80:844866.

Jacobs HI, Hopkins DA, Mayrhofer HC, Bruner E, van Leeuwen FW, Raaijmakers W, Schmahmann JD. The cerebellum in Alzheimer's disease: evaluating its role in cognitive decline. Brain 2018;141:37-47.

Larsen JO, Skalicky M, Viidik A. Does long-term physical exercise counteract age-related Purkinje cell loss A stereological study of rat cerebellum. J Comp Neurol 2000;428:213-222.

Lee JM, Kim CJ, Park JM, Song MK, Kim YJ. Effect of treadmill exercise on spatial navigation impairment associated with cerebellar Purkinje cell loss following chronic cerebral hypoperfusion. Mol Med Rep 2018; 17:8121-8128.

Lee JM, Park JM, Song MK, Oh YJ, Kim CJ, Kim YJ. The ameliorative effects of exercise on cognitive impairment and white matter injury from blood-brain barrier disruption induced by chronic cerebral hypoperfusion in adolescent rats. Neurosci Lett 2017;638:83-89.

Marambaud P, Dreses-Werringloer U, Vingtdeux V. Calcium signaling in neurodegeneration. Mol Neurodegener 2009;4:20.

Marques-Aleixo I, Santos-Alves E, Balca M, Rizo-Roca D, Moreira P, Oliveira P, Ascensão A. Physical exercise improves brain cortex and cerebellum mitochondrial bioenergetics and alters apoptotic, dynamic 
and auto(mito)phagy markers. Neuroscience 2015;301:480-495.

Martin LA, Escher T, Goldowitz D, Mittleman G. A relationship between cerebellar purkinje cells and spatial working memory demonstrated in a lurcher/chimera mouse model system. Genes Brain Behav 2004;3: 158-166.

Palesi F, De Rinaldis A, Castellazzi G, Calamante F, Muhlert N, Chard D, Tournier D, Magenes G, D’Angelo E, Wheeler-Kingshott CAG. Contralateral cortico-ponto-cerebellar pathways reconstruction in humans in vivo: implications for reciprocal cerebro-cerebellar structural connectivity in motor and non-motor areas. Sci Rep 2017;7:12841.

Pchitskaya E, Popugaeva E, Bezprozvanny I. Calcium signaling and molecular mechanisms underlying neurodegenerative diseases. Cell Calcium 2018;70:87-94.

Schefer V, Talan MI. Oxygen consumption in adult and AGED C57BL/6J mice during acute treadmill exercise of different intensity. Exp Gerontol 1996;31:387-392.

Schmahmann JD, Sherman JC. The cerebellar cognitive affective syndrome. Brain 1998;121(Pt4):561-579.

Seo JH, Park HS, Park SS, Kim CJ, Kim DH, Kim TW. Physical exercise ameliorates psychiatric disorders and cognitive dysfunctions by hippocampal mitochondrial function and neuroplasticity in post-traumatic stress disorder. Exp Neurol 2019;322:113043.

Seo TB, Kim BK, Ko IG, Kim DH, Shin MS, Kim CJ, Yoon JH, Kim H. Effect of treadmill exercise on Purkinje cell loss and astrocytic reaction in the cerebellum after traumatic brain injury. Neurosci Lett 2010;481: 178-182.

Sui R, Zhang L. Cerebellar dysfunction may play an important role in vascular dementia. Med Hypotheses 2012;78:162-165.

Tomlinson SP, Davis NJ, Morgan HM, Bracewell RM. Cerebellar contributions to spatial memory. Neurosci Lett 2014;578:182-186.

Tse AC, Wong TW, Lee PH. Effect of low-intensity exercise on physical and cognitive health in older adults: a systematic review. Sports Med Open 2015;1:37.

Venkat P, Chopp M, Chen J. Models and mechanisms of vascular dementia. Exp Neurol 2015;272:97-108.

Welsh JP, Yuen G, Placantonakis DG, Vu TQ Haiss F, O'Hearn E. Molliver ME, Aicher SA. Why do Purkinje cells die so easily after global brain ischemia? Aldolase C, EAAT4 and the cerebellar contribution to posthypoxic myoclonus. Adv Neurol 2002;89:331-359.

Zhao S, Chen N, Yang Z, Huang L, Zhu Y, Guan S, Chen Q, Wang JH. Ischemia deteriorates the spike encoding of rat cerebellar Purkinje cells by raising intracellular Ca2+. Biochem Biophys Res Commun 2008;366:401-407.

Zhivotovsky B, Orrenius S. Calcium and cell death mechanisms: a perspective from the cell death community. Cell Calcium 2011;50:211-221.

Zorov DB, Juhaszova M, Sollott SJ. Mitochondrial reactive oxygen species (ROS) and ROS-induced ROS release. Physiol Rev 2014;94:909-950. 